\title{
Proton Momentum Distribution in a Protein Hydration Shell
}

\author{
R. Senesi and A. Pietropaolo \\ Dipartimento di Fisica and Centro NAST, Università degli Studi di Roma “Tor Vergata,” Via della Ricerca Scientifica 1, \\ 00133 Roma, Italy \\ A. Bocedi, S. E. Pagnotta, and F. Bruni* \\ Dipartimento di Fisica “E. Amaldi," Università degli Studi di Roma Tre, Via della Vasca Navale 84, 00146 Roma, Italy
}

(Received 7 November 2006; published 27 March 2007)

\begin{abstract}
The momentum distribution of protons in the hydration shell of a globular protein has been measured through deep inelastic neutron scattering at 180 and $290 \mathrm{~K}$, below and above the crossover temperature $T_{c}=1.23 T_{g}$, where $T_{g}=219 \mathrm{~K}$ is the glass transition temperature. It is found that the mean kinetic energy of the water hydrogens shows no temperature dependence, but the measurements are accurate enough to indicate a sensible change of momentum distribution and effective potential felt by protons, compatible with the transition from a single to a double potential well. This could support the presence of tunneling effects even at room temperature, playing an important role in biological function.
\end{abstract}

The water molecules that make up the hydration shell in the immediate vicinity of a protein surface are particularly relevant for processes ranging from enzymatic activity to anhydrobiosis. The dynamical properties of these water molecules, closely resembling that of a glassy system $[1,2]$, and, in particular, the interplay with the slaved dynamical behavior of the protein itself, has likely implications for biological functionality [3-6], and as such is a subject of active research. The accurate study of the properties of water molecules hydrating proteins has seen a resurgence of interest in the past few years, possibly due to the availability of powerful simulation techniques along with the advent of new experimental approaches. One of the best studied examples of the interplay between hydration water and protein behavior is represented by the globular enzyme lysozyme, a protein-water system of particular interest due to the presence, at room temperature, of a percolative transition of the conductivity at a critical hydration level coinciding with the onset of enzymatic activity [7]. This transition is due to proton displacements along hydrogen-bonded water molecules adsorbed on the protein surface, with ionizable groups as sources of migrating protons [8]. The dynamics of these migrating protons shows interesting analogies with the dynamical behavior of fragile proton glasses, the electric counterparts of magnetic spin glasses $[9,10]$. In particular, the nonexponential behavior of the relaxation function is well accounted by the well known Kohlrausch-Williams-Watts (KWW) equation $\phi(t)=\exp \left[-\left(t / \tau_{\mathrm{KWW}}\right)^{\beta_{\mathrm{KWw}}}\right], \tau_{\mathrm{KWW}}$ being a characteristic time scale and $\beta_{\mathrm{KWW}}$ the nonexponentiality parameter. It has been suggested [11] that $\beta_{\mathrm{KWW}}(T)$ plays the role of an universal parameter for a wide array of glassy systems, especially with respect to its temperature dependence. Namely, $\beta_{\mathrm{KWW}}(T)$ shows a crossover from a low temperature region, where it increases with temperature, to a high temperature region where it is essentially temperature independent. The crossover temperature for lysozyme has been found equal to $T_{c}=1.23 T_{g}$, where $T_{g}=219 \mathrm{~K}$ is the glass transition temperature, defined as the temperature where the dielectric relaxation time is $100 \mathrm{~s}$ [12]. Figure 1 (adapted from Ref. [12]) shows our recent experimental determination of $\beta_{\mathrm{KWW}}$, for a sample of hydrated lysozyme. In particular, note that deviation from $\beta_{\mathrm{KWW}}=$ 1 below $T_{c}$ is a signature of a pronounced slow down in dynamical processes characterized by the relaxation time $\tau_{\mathrm{KWw}}$, which is believed to be related to spatial heterogeneity in the dynamics [13]. Within this framework, it is

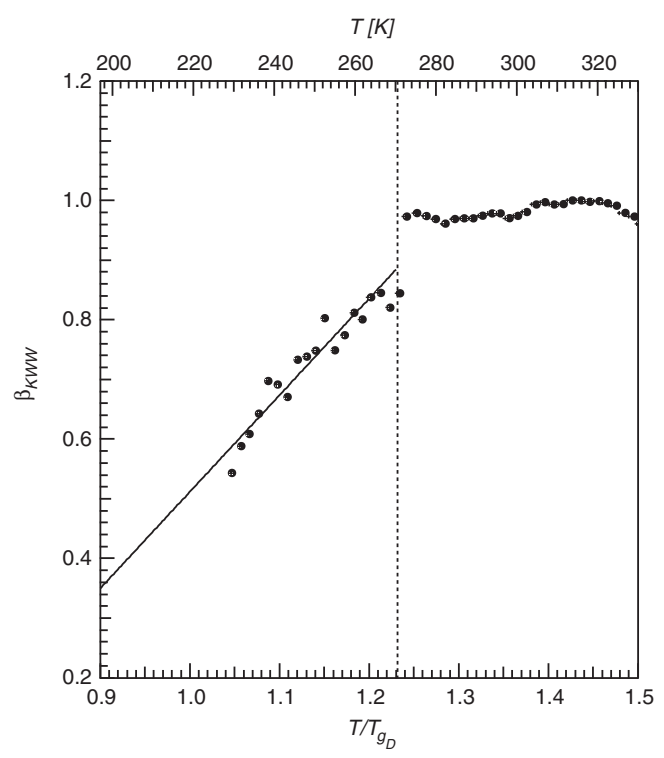

FIG. 1. Temperature dependence of the nonexponentiality parameter $\beta_{\mathrm{KWW}}$. The solid line is a linear fit of the data in the interval $(1.0,1.2)$. The dashed vertical line is drawn at $T=$ $1.23 T_{g_{D}}$ representing the crossover temperature between two distinct dynamical regimes. 
conceivable that the dynamics of water protons in the first hydration shell, governed by solvent fluctuations [14,15], should be different below and above the crossover temperature $T_{c}$. In other words, momentum distribution of water protons, $n(p)$, should display a marked temperature dependence. The development of pulsed neutron sources allows the direct measurement of proton momentum distribution $[16,17]$, through the deep inelastic neutron scattering (DINS) technique. The high energy and wave vector transfers achieved in DINS experiments, allow us to describe the scattering process within the framework of the impulse approximations (IA) $[17,18]$. In the IA, the dynamical structure factor is related to the $n(p)$ through the relation:

$$
S_{\mathrm{IA}}(\vec{q}, \omega)=\int n(\vec{p}) \delta\left(\omega-\frac{\hbar q^{2}}{2 M}-\frac{\vec{p} \cdot \vec{q}}{M}\right) d \vec{p},
$$

where $\hbar \omega$ is the energy transfer, $\frac{\hbar^{2} q^{2}}{2 M}$ is the recoil energy of the struck atom of mass $M$, and $\vec{q}$ is the wave vector transfer. This scattering law can be then expressed in terms of a scaling function: $J_{\mathrm{IA}}(y, \hat{q})=\frac{\hbar q}{M} S_{\mathrm{IA}}(\vec{q}, \omega)$, where $y=$ $\frac{M}{\hbar q}\left(\omega-\frac{\hbar q^{2}}{2 M}\right)$ is the West scaling variable [17]. The function $J_{\mathrm{IA}}(y, \hat{q})$, often referred to as the neutron compton profile (NCP) [17], represents the probability density distribution of $y$, the atomic momentum component along the direction of the wave vector transfer $\hat{q}$. In an isotropic system there is no dependence on $\hat{q}$, and the response function is given by $J_{\mathrm{IA}}(y)=2 \pi \int_{|y|}^{\infty} p n(p) d p$, resulting in

$$
n(p)=-\frac{1}{2 \pi p}\left(\frac{\partial J(y)}{\partial y}\right)_{y=p} .
$$

For finite $q$ values, deviation from IA can be accounted for in terms of additive corrections to the asymptotic form $J(y, q)=J_{\mathrm{IA}}(y)+\Delta J(y, q)$ [17]. The DINS experiment on the lysozyme sample has been carried out on the VESUVIO spectrometer [17], at the ISIS spallation neutron source (Rutherford Appleton Laboratory, U.K.). The lysozyme powder samples were prepared as described in Ref. [10]. Measurements were made at 180 and $290 \mathrm{~K}$, above and below the crossover temperature $T_{c}$. Experimental data at both temperatures have been acquired in the standard resonance filter configuration, employing the single difference (SD) and the double difference (DD) methods [19], the latter providing a narrow instrumental resolution, with the aim of carrying out a detailed line shape analysis of the proton NCP. Standard data reduction procedures have been applied to obtain the fixed-angle experimental NCP, $F(y, \phi)=J(y, \phi) \otimes R(y, \phi)$ [17,20], $R(y, \phi)$ being the fixed-angle spectrometer resolution. The latter has been evaluated by a Monte Carlo simulation [20]. Data have been also rebinned onto constant- $q$ spectra with a width $\Delta q$ of approximately $4 \AA^{-1}$ (as done in Ref. [21]) to obtain $F(y, q)$ and the corresponding resolution functions. The momentum distribution line shape analysis consists in a simultaneous fitting of SD and DD $F(y, q)$ spectra, employing a resolution-broadened modelindependent line shape function for $J(y, q)$ :

$$
J(y, q)=\frac{e^{-\left(y^{2} / 2 \sigma^{2}\right)}}{\sqrt{2} \bar{\pi} \sigma}\left[\sum_{n} \frac{c_{n}}{2^{n} n !} H_{2 n}\left(\frac{y}{\sqrt{2} \sigma}\right)+\frac{k}{q} H_{3}\left(\frac{y}{\sqrt{2} \sigma}\right)\right]
$$

where $H_{n}$ are Hermite polynomials and $\sigma, c_{n}, k$ are fitting parameters. In particular, the parameter $k$ accounts for the finite- $q$ corrections $\Delta J(y, q)$ [16]. Using Eq. (2), the momentum distribution can be expressed as [16]

$$
n(p)=\frac{e^{-\left(p^{2} / 2 \sigma^{2}\right)}}{(\sqrt{2 \pi} \sigma)^{3}} \sum_{n} c_{n}(-1)^{n} L_{n}^{1 / 2}\left(\frac{p}{\sqrt{2} \sigma}\right)
$$

where $L_{n}^{1 / 2}$ are generalized Laguerre polynomials. The series have been truncated to order $n=3$, being higher order terms insignificant for the fitting procedures. The same procedure has been applied also to fixed-angle data, yielding the same set of parameters within errors, ensuring an important internal check of data analysis consistency. Figure 2 shows the experimental response function $F(y, q)$ of hydrated lysozyme at $T=180 \mathrm{~K}$, for $q=57 \AA^{-1}$, with the corresponding best fit.

Figure 3 shows proton momentum distributions at $T=$ 180 and $290 \mathrm{~K}$. The standard deviation of these distributions $\sigma$ is related to the mean kinetic energy $\left\langle E_{k}\right\rangle$ through the relation $\sigma^{2}=\frac{2 M}{3 \hbar^{2}}\left\langle E_{k}\right\rangle$. The $\left\langle E_{k}\right\rangle$ calculated according to this definition are $215.3 \pm 1.5 \mathrm{meV}$ and $216.1 \pm 4.4 \mathrm{meV}$ at 180 and $290 \mathrm{~K}$, respectively. Quite unexpectedly, there is

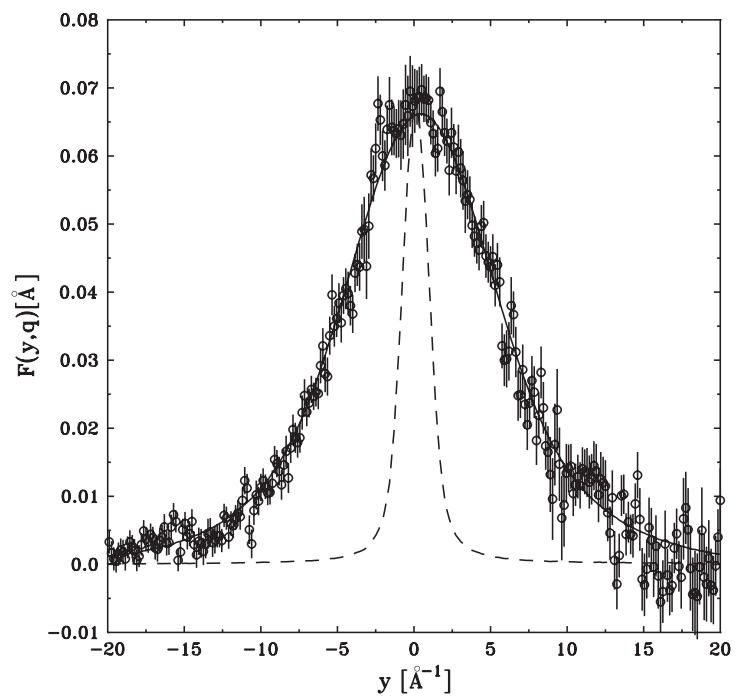

FIG. 2. Neutron Compton profile, $F(y, q)$, of hydrated lysozyme at $T=180 \mathrm{~K}$, for $q=57 \pm 4 \AA^{-1}$, acquired with single difference method (circles with error bars); best fit (solid line); spectrometer resolution, $R(y, q)$, dashed line. Other constant- $q$ data employed were $37 \pm 3 \AA^{-1}, 40 \pm 4 \AA^{-1}, 46 \pm 4 \AA^{-1}$. The lysozyme sample was prepared at $p \mathrm{H} 7$ and at hydration level $h=0.26 \mathrm{~g} / \mathrm{g}$ (grams of water over grams of dry protein). 


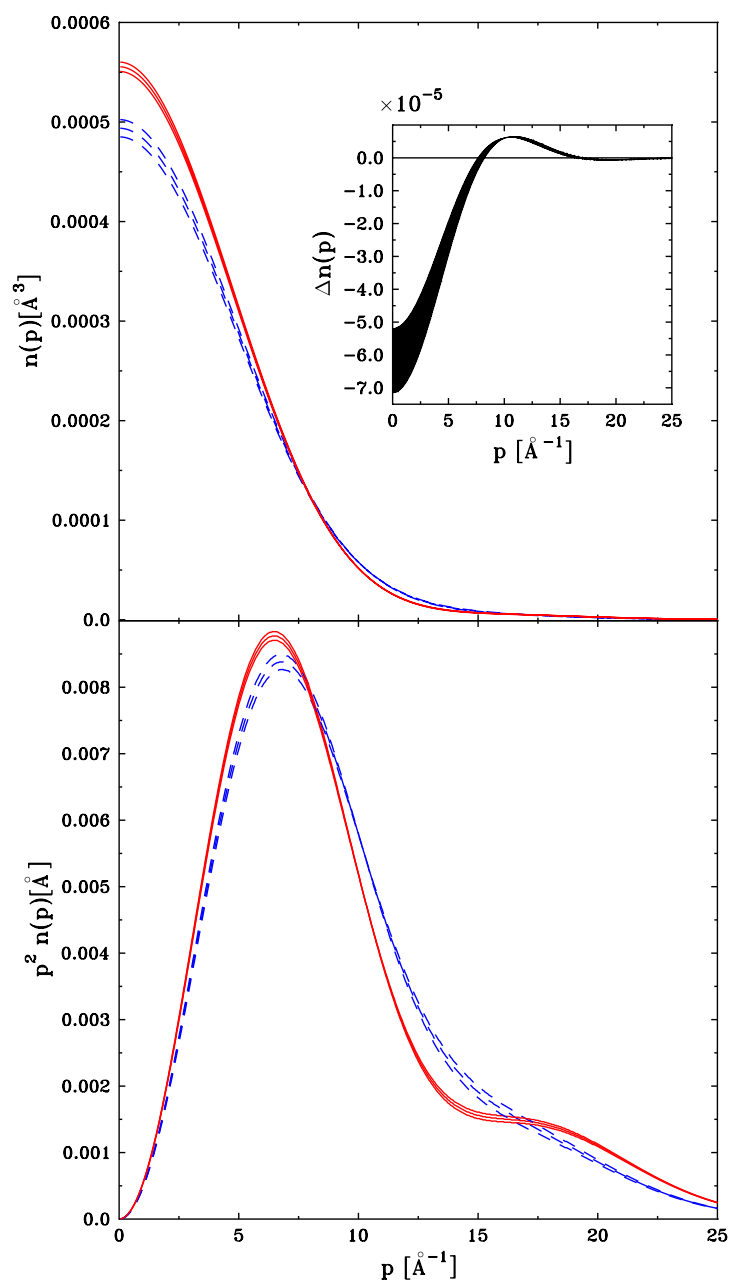

FIG. 3 (color online). Upper panel: proton momentum distribution of hydrated lysozime at $T=290 \mathrm{~K}$ (solid line) and $T=$ $180 \mathrm{~K}$ (dashed line). Upper and lower lines delimit errors associated to $n(p)$ (see Ref. [16] for details). The inset shows the momentum distribution difference $\Delta n(p)$ between lower and higher temperature data. Lower panel: spherically averaged momentum distributions, $p^{2} n(p)$, of hydrated lysozime at $T=$ $290 \mathrm{~K}$ (solid line) and $T=180 \mathrm{~K}$ (dashed line).

no temperature dependence, beside the small $k_{B} T$ contribution, on these energy values, being identical within the experimental uncertainty. Moreover, these $\left\langle E_{k}\right\rangle$ values are larger than that reported for bulk water at $296 \mathrm{~K}$ [17], namely, $145 \mathrm{meV}$. This result might look counterintuitive at first glance, nevertheless we recall that water in the hydration layer of a globular protein can be considered as confined, in terms of restricted geometry, and interacting with the surface. The loss of tetrahedral ordering of water due to confinement [22] should then lead to a higher mean kinetic energy, as indicated by DINS experiment on water, at supercritical temperature and pressure, confined in the interstices of $\mathrm{C}_{60}$ polycrystalline powder [23], and water confined at ambient temperature and pressure in a porous silica matrix [24]. In particular, confining water in a hydrophilic silica matrix with pore diameters of about the same size of that of the globular lysozyme, yields quite similar $\left\langle E_{k}\right\rangle$ values [24]. To highlight the differences between the $n(p)$ measured at the two temperatures, we have plotted the spherically averaged momentum distribution $p^{2} n(p)$ (Fig. 3, lower panel). In particular, $p^{2} n(p)$ at $290 \mathrm{~K}$ shows a low-momentum narrowing along with a shoulder at about $p=17 \AA^{-1}$, in analogy with the above mentioned DINS experiments on confined water. This shoulder could be interpreted as being due to a small population of protons with unusually high momentum and highly localized, or, alternatively and less unphysically, in terms of a single particle in an effective potential due to its neighbors [23]. The narrowing at low $p$ and the shoulder at large $p$ have been attributed, in the case of other hydrogen-bonded systems, to coherent interference of the proton over the two sites of a double-well potential $[25,26]$. Namely, with this latter interpretation, and assuming that each water proton is surrounded by a covalent bound oxygen and a hydrogen-bonded oxygen, the proton is coherent over two separated sites along the hydrogen bond. Before discussing this issue further, we would like to offer an alternative interpretation of the DINS data due to the simultaneous recoil of water and protein hydrogens. Actually, we have no way in our experiments to distinguish between water and protein hydrogens, thus the measured $n(p)$ shows the contribution of both types of $\mathrm{H}$ atoms. However, careful molecular dynamics (MD) studies, capable to separate the effects of protein potential surface and of solvent mobility on the atomic fluctuations, have shown that it is mainly solvent mobility that determines the magnitudes of protein fluctuations not only at the protein surface but also in the protein core at and above $180 \mathrm{~K}$ [3]. In particular, motions of protein hydrogens have been investigated by means of quasielastic scattering on the same lysozyme sample [27], showing that the onset of anharmonicity, assigned to methyl group rotation, appears around $T=100 \mathrm{~K}$ even for a dry sample. Nevertheless, in the same article, a much larger dynamical transition, clearly induced by water in the hydration shell at about $T=200 \mathrm{~K}$, is observed at a hydration level $h$ greater than $0.2 \mathrm{~g} / \mathrm{g}$ (recall our sample is at $h=0.26 \mathrm{~g} / \mathrm{g}$ ). This experimental result is consistent with the simulation described in [3], confirming that solvent dynamics is slaving that of the protein and that, even if protein hydrogen dynamics is activated at $100 \mathrm{~K}$, its contribution is negligible compared to that of water hydrogens in the protein hydration shell at higher temperature. We can therefore assume that the temperature dependence of $n(p)$ is mainly due to water hydrogens, with a momentum distribution of protein hydrogens essentially independent of temperature [i.e., showing the same proton dynamics on the time scale of the DINS experiment $\left.\left(10^{-15}-10^{-16} \mathrm{~s}\right)\right]$. To a first approximation, since the proton is surrounded by much heavier oxygen atoms, the proton momentum distribution can be thought of as arising from its confinement in the potential well provided by the oxygens. These can be regarded as fixed in positions, and the interaction with other protons will modify the potential well [23]. The 
presence of the shoulder at $p=17 \AA^{-1}$ in the $p^{2} n(p)$, along with its absence at $180 \mathrm{~K}$, would then suggest a modification of the confining potential compatible with the transition from a single well at lower temperature to a double-well potential at $290 \mathrm{~K}$. Interestingly, MD studies [3,4] and neutron scattering experiment [28], gave evidence, albeit on a quite different time scale, of a transition, largely due to the solvent dynamics, from harmonic to anharmonic dynamics in globular proteins as a function of increasing temperature. To further corroborate the hypothesis regarding the transition from a single to a doublewell potential, the inset in the upper panel of Fig. 3 shows the difference between the $n(p)$ at the two temperatures investigated. Oscillations are visible and their absolute value, over the entire $p$ range, is larger than the uncertainty deriving from the fitting procedures described above. It should be noted that oscillations maxima and minima occur at about $p=10$ and $20 \AA^{-1}$, corresponding to 4 and 8 times the quantity $2 \pi / d$. Remarkably, the distance $d$ can be then calculated to be $2.5 \AA$, in good agreement with the average oxygen-oxygen distance of two nearby water molecules adsorbed on the protein surface [29], leading to an hydration shell denser than the bulk water. The dynamical transition driven by water around $200 \mathrm{~K}[3,4]$ could be responsible of the reduced distance between hydration water oxygens, thus changing the local structure around protons. This appears to induce the transition of the potential felt by protons towards a flattened-bottom or lowbarrier double well. The measured momentum distribution reflects the delocalization of protons over two sites along the hydrogen bond, representing a signature of a tunneling effect [30].

In summary, we have used DINS to measure the momentum distribution of protons in the hydration shell of a globular protein above and below the crossover temperature $T_{c}$. Here we are dealing with a disordered system and, in particular, on its dynamical properties measured on a very short time scale. This approach provides new information, not accessible with other experimental techniques, that could in turn stimulate simulation studies on the same, and often neglected, time scale. Confining water in the hydration shell of the protein increases the proton mean kinetic energy to a value above that of the bulk water phase. The $n(p)$ can be measured with sufficient accuracy to allow a comparison between the data collected at the two temperatures investigated. To a first approximation, the $n(p)$ can be thought of as arising from the confinement of the proton in the potential well provided by water oxygens in the protein hydration shell, with a oxygen-oxygen first neighbor distance smaller that the corresponding distance in the bulk phase. This could support the presence of tunneling effects even at room temperature [31], playing an important role in the biological function [32,33].

This work was supported within the CNR-CCLRC Agreement No. 01/9001 concerning collaboration in scientific research at the spallation neutron source ISIS. The financial support of the Consiglio Nazionale delle Ricerche in this research is hereby acknowledged.

*Author to whom correspondence should be addressed. Email address: bruni@ fis.uniroma3.it

[1] M. Tarek and D. J. Tobias, Biophys. J. 79, 3244 (2000).

[2] A. R. Bizzarri and S. Cannistraro, J. Phys. Chem. B 106, 6617 (2002).

[3] D. Vitkup et al., Nat. Struct. Biol. 7, 34 (2000).

[4] A. L. Tournier and J. C. Smith, Phys. Rev. Lett. 91, 208106 (2003).

[5] P. W. Fenimore et al., Proc. Natl. Acad. Sci. U.S.A. 101, 14408 (2004).

[6] T. Becker et al., Biophys. J. 87, 1436 (2004).

[7] J. A. Rupley and G. Careri, Adv. Protein Chem. 41, 37 (1991).

[8] G. Careri, Prog. Biophys. Molec. Biol. 70, 223 (1998).

[9] F. Pizzitutti and F. Bruni, Phys. Rev. E 64, 052905 (2001).

[10] F. Bruni and S. E. Pagnotta, Phys. Chem. Chem. Phys. 6, 1912 (2004).

[11] A. Alegria et al., Phys. Rev. E 59, 6888 (1999); L. Angelani et al., Phys. Rev. Lett. 81, 4648 (1998).

[12] S. E. Pagnotta et al., Phys. Rev. E 71, 031506 (2005).

[13] P. G. Debenedetti and F.H. Stillinger, Nature (London) 410, 259 (2001).

[14] D. Marx et al., Nature (London) 397, 601 (1999).

[15] M. Peyrard, Phys. Rev. E 64, 011109 (2001).

[16] G. F. Reiter, J. Mayers, and J. Noreland, Phys. Rev. B 65, 104305 (2002).

[17] C. Andreani et al., Adv. Phys. 54, 377 (2005).

[18] G. Reiter and R. Silver, Phys. Rev. Lett. 54, 1047 (1985).

[19] C. Andreani et al., Nucl. Instrum. Methods Phys. Res., Sect. A 497, 535 (2003).

[20] J. Mayers, A. L. Fielding, and R. Senesi, Nucl. Instrum. Methods Phys. Res., Sect. A 481, 454 (2002); C. Andreani, C. Pantalei, and R. Senesi, J. Phys. Condens. Matter 18, 5587 (2006).

[21] K. H. Andersen, W. G. Stirling, and H. R. Glyde, Phys. Rev. B 56, 8978 (1997).

[22] F. Bruni, M. A. Ricci, and A. K. Soper, J. Chem. Phys. 109, 1478 (1998); A. K. Soper, F. Bruni, and M. A. Ricci, J. Chem. Phys. 109, 1486 (1998).

[23] G. F. Reiter et al., Braz. J. Phys. 34, 142 (2004).

[24] V. Garbuio et al. (unpublished).

[25] G. F. Reiter, J. Mayers, and P. Platzman, Phys. Rev. Lett. 89, 135505 (2002).

[26] G. Reiter et al., Phys. Rev. Lett. 97, 247801 (2006).

[27] J. H. Roh et al., Phys. Rev. Lett. 95, 038101 (2005).

[28] V. Réat et al., Proc. Natl. Acad. Sci. U.S.A. 97, 9961 (2000).

[29] F. Merzel and J. C. Smith, Proc. Natl. Acad. Sci. U.S.A. 99, 5378 (2002).

[30] J. Lobaugh and G. A. Voth, J. Chem. Phys. 104, 2056 (1996).

[31] M.E. Tuckerman and D. Marx, Phys. Rev. Lett. 86, 4946 (2001).

[32] W. J. Bruno and W. Bialek, Biophys. J. 63, 689 (1992).

[33] D. Antoniou and S. D. Schwartz, J. Phys. Chem. B 105, 5553 (2001). 